\title{
Referee Acknowledgements
}

The individuals listed below generously contributed their time and expertise to ACES this year. I thank each of them for doing an excellent job in reviewing in a timely manner the manuscripts they received. Their assistance has been invaluable to Comparative Economic Studies. THANKS!

Hans Aage

Jan Adam

Michael Alexeev

Christine Amsler

Donna Bahry

Daniel Berkowitz

Milica Bookman

Michael L. Boyd

Kenneth Boyer

Byron Brown

William A. Byrd

Robert W. Campbell

Richard L. Carson

Elizabeth Clayton

Irwin L. Collier

Laszlo Csaba

Robert Dernberger

John F. Elliott

Michael Ellman

Zbigniew Fallenbuchl

Shirley Gedeon

Harry Holzer

Martin Houda

Holland Hunter

Barry Ickes

Marvin Jackson

Barbara Katz

Michael Keren

Anthony Koo

Konrad Kubin

Fyodor I. Kushnirsky

Nicholas Lardy

Kazimierz Laski

Carl Liedholm
Cyril Lin

Mark Lundell

Paul Marer

Michael Marrese

Carl H. McMillan

Deborah Milenkovitch

James R. Millar

Janet Mitchell

William Moskoff

Peter Murrell

Egon Neuberger

Rowena Pecchenino

Dwight Perkins

Joyce Pickersgill

Richard Portes

Frederic L. Pryor

Louis Putterman

Ryszard Rapacki

Robert Rasche

Michal Rutkowsky

Tsuneaki Sato

Mark Schaffer

Gertrude Schroeder

Catherine Sokil

Robert C. Stuart

Tomas Tepper

Judith Thornton

Peter Toumanoff

Vittorio Valli

Don A. Van Atta

Martin Weitzman

Robert Whitesell

Huizhong Zhou

Andrew Zimbalist 